\title{
TIBULO A TRAVÉS DE OVIDIO
}

This paper analyses an unusual feature of Tibulo's language and versification: the repeated use of the perfect infinitive of the type continuisse in the second hemistich of the pentameter. Ovidio cleverly understood its stylistic value and very skilfully used it when he wanted to homage the elegiac poet.

1. Releyendo la elegía IX del libro tercero de los Amores ovidianos me llama la atención la gran frecuencia con que el poeta recurre a pentasílabos terminados en -isse $(t)$ para ocupar la parte que precede al bisílabo final del pentámetro:
v. 22: carmine quid uictas obstipuisse feras
v. 24: dicitur inuita concinuisse lyra
v. 34: sistra? quid in uacuo secubuisse toro?
v. 46: sunt quoque qui lacrimas continuisse negant
v. 48: ignotum uili supposuisset humo

Nada menos que cinco pentámetros de los treinta y cuatro de que consta el poema, es decir, el 14,70 por 100 , presentan en su segundo hemistiquio esta disposición.

No hay otra composición en el libro que se le asemeje en este punto: las que más parecen aproximarse son la elegía seis, con tres casos (que sólo suponen el 5,66 por 100 de los cincuenta y tres pentámetros) y la siete, con cuatro casos, que constituyen el 9,52 por 100 .

Este porcentaje en el conjunto del libro es del 4,59 por 100 (20 pentámetros entre 435); en el libro I, 1,82 por 100 (siete casos entre 384 ) y en el segundo, el 3,20 por 100 (trece, entre 406 pentámetros). Del total de los pentámetros de Amores (1225) sólo 40, el 3,26 por 100 presentan un pentasílabo de este tipo.

$\mathrm{Y}$ en el resto de la obra ovidiana, las cosas no parecen ser muy diferentes: así, por ejemplo, sólo siete (1,93 por 100) de los 362 pentámetros de Fastos I son de este tipo; en los 462 de Pónticas IV sólo hemos encontrado cinco $(1,08$ por 100). 
Uniendo estas muestras de la producción media y final de Ovidio a los pentámetros juveniles de Amores, se constituye un corpus de 2.049 versos, entre los cuales los del tipo que venimos comentando son cincuenta y dos, es decir, el 2,54 por 100.

La singularidad de Amores III 9 es, por tanto, más que evidente y provoca la curiosidad del lector que la ha advertido: ¿por qué aquí esta insistencia del poeta en semejantes palabras, cuando en ningún otro pasaje de esta obra $y$, quizá, de ninguna otra a lo largo y ancho de su vasta producción en dísticos elegíacos ocurre otro tanto?

2. Lo primero, por supuesto, ante esta cuestión es acudir a otros poetas y comprobar su comportamiento al respecto. $\mathrm{Y}$, cosa que no suele ocurrir con mucha frecuencia, no tarda en surgir una posible respuesta.

En efecto, un repaso a los pentámetros de Catulo, Tibulo (los libros I y II y el corpus Tibullianum ${ }^{1}$ ), Propercio y Marcial, apunta en seguida la singularidad de Tibulo (los libros I y II) frente a todos los demás en lo que se refiere al empleo de este tipo de pentasílabos en el segundo hemistiquio del pentámetro (en adelante, pentámetros tipo $\mathrm{A}$ ):

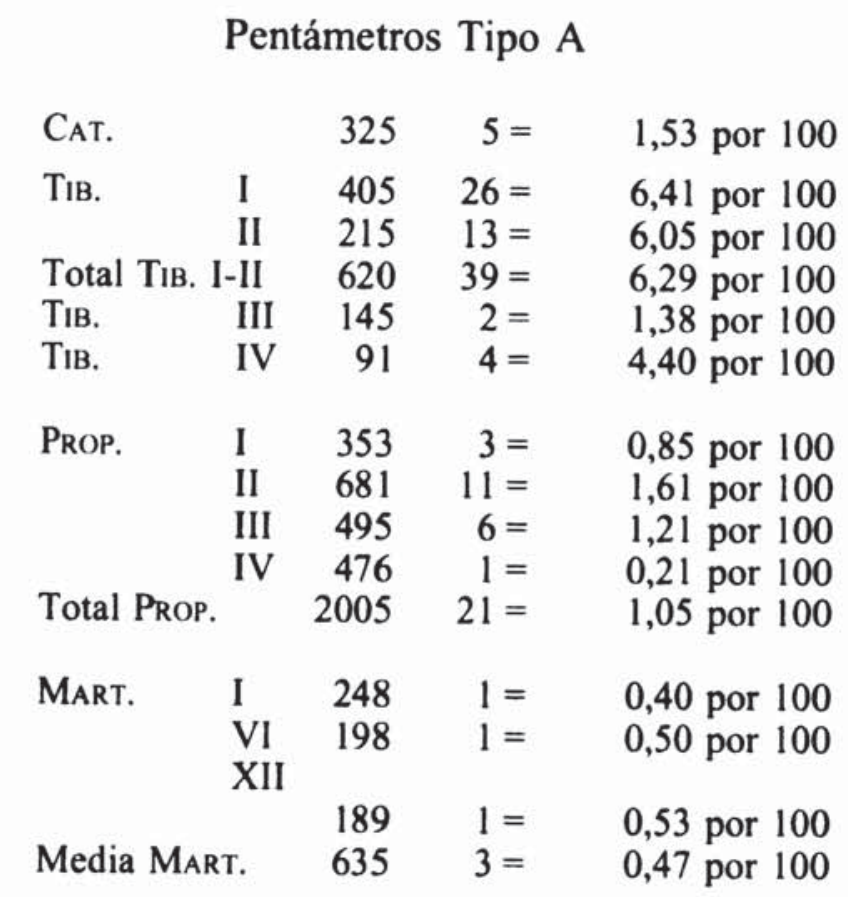

Basta una ojeada a la anterior estadística para comprobar que la diferencia entre Tibulo y todos los demás, incluido el propio Ovidio, es clara. La tendencia a colocar una forma verbal (infinitivo de pasado, sobre todo, aunque tam-

1 Las Lygdami elegiae de III 1-6 y los dísticos del libro IV: 2-6 De Sulpicia incerti auctoris elegiae, 7-12 Sulpiciae elegidiae y 13-14 Incerti auctoris carmina. 
bién pluscuamperfecto de subjuntivo) pentasilábica ante el bisílabo final del pentámetro parece, pues, un rasgo típico de este poeta.

Que así es parece indicarlo también la diferencia existente entre el auténtico Tibulo, los libros I y II y el resto del corpus, sobre todo, el libro III. El libro IV, con un 4,4 por 100 , sería junto a Amores III, con un 4,59 por 100 el que más se le aproximaría.

Que así es lo sugieren asimismo bastantes elegías, si se las considera por separado. Así, por ejemplo, en I 5,10 y 12 encontramos dos pentámetros de este tipo seguidos:

ille ego cum tristi morbo defessa iaceres
te dicor uotis eripuisse meis;
ipseque te circum lustraui sulpure puro,
carmine cum magico praecinuisset anus;

En I 2 estos pentámetros representan el 6,12 por 100 ; en II 1, el 6,67 por 100 ; en I 4 y en I 6 , el 6,98 por 100 ; en I 1 , el 7,7 por 100 ; en II 5 , el 9,83 por 100; en I 8 aparece este tipo A nada menos que en cinco de los treinta y nueve pentámetros $(12,82$ por 100$)$, tres de ellos $(8,10$ y 12), además, seguidos:

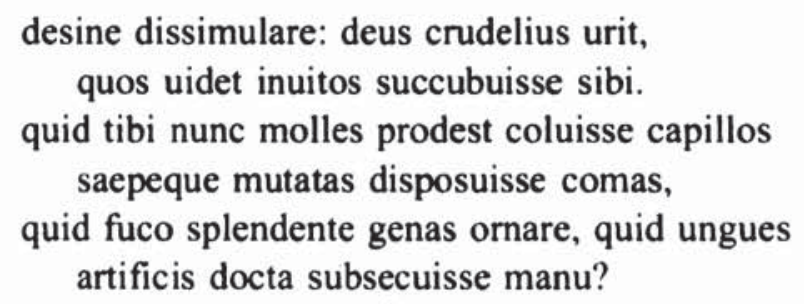

He aquí, pues, un rasgo definidor, una marca del pentámetro tibuliano.

3. He aquí a la vez una prueba de la sutileza de Ovidio como versificador, ya que supo reconocer dicho rasgo y tuvo el acierto de potenciarlo en el momento de escribir una elegía (Amores III 9) a la muerte de Tibulo.

"On the death of Tibullus, he wrote an elegy which bears testimony to the most sympathetic intimacy with Tibullus' writings», escribió hace años Frän$\mathrm{kel}^{2}$. Así lo reconoce más tarde Taylor ${ }^{3}$, para quien esta íntima familiaridad de Ovidio con Tibulo se detecta particularmente «in verbal echoes and also, to some extent, in metrics and style".

Para Taylor, la comparación de la técnica es, en general, «unrewarding», aunque encuentra dos cuestiones que sí merece la pena mencionar: una, el hecho de que Ovidio en $\mathrm{Am}$. III 9 evite por completo el empleo de $o$ con

${ }^{2}$ H. Fraenkel, Ovid. A poet between two worlds, Berkeley-Los Ángeles 1945 (=1956), p. 10.

3 J. H. Taylor, «Amores 3, 9: A Farewell to Elegy», Latomus 29, 1970, p. 475 ss. 
vocativo (al que Tibulo nunca recurre), cuando en el resto de la obra es de uso común (quince veces en Amores y cinco en el libro tercero). La otra se refiere al empleo de e/ex y $a / a b$ : Tibulo siempre pone $e, a$ ante consonante y $e x, a b$ ante vocal, excepto una vez, en ex uirgis. Ovidio, en cambio, no parece muy preocupado por esta distinción; sin embargo, en III 9,40 encontramos $e$ toto, a pesar de que ex es más común en Amores, y ex uero (III 9,4), paralelo a la única excepción, ex uirgis, de Tibulo.

La imitación donde Taylor la ve más claramente es en el léxico, en particular en los siguientes pasajes:

III 9, 53-54 iunxerunt... oscula puede ser un eco de Tib. I 1, 69 iungamus amores. III 9, 58 moriens deficiente manu es una cita literal de Tib. I 1, 60.

III 9, 33 quid... prosunt (III 9, 21 quid... profuit) es una expresión favorita de Tibulo. III 9, 34 secubuisse toro ${ }^{4}$ refleja a Tib. I 3, 26.

III 9, 25 pius cole sacra se halla probablemente inspirado en Tib. I 3, 25 pie dum sacra colis.

III 9, 33-36 tiene reminiscencias de Tib. I 3, 23-26.

III 9, 47-48 puede ser un reflejo de Tib. I 3, 3.

En III 9, 50-52 las figuras de la madre y de la hermana se corresponden con las de Tib. I 3, 5-8.

III 9, 6 ardet in exstructo corpus inane rogo se halla posiblemente en conexión con Tib. Il $4,46^{5}$.

Con estos y otros pasajes queda claro para Taylor que la imitación la ha centrado aquí Ovidio principalmente en la «verbal evidence».

Se le plantea entonces a la autora la pregunta de por qué Ovidio no ha tratado de llevar a cabo una imitación técnica más sutil. Y sugiere para ello dos posibles explicaciones: la primera, que muchos rasgos estilísticos de $\mathrm{Ti}$ bulo habían sido tomados, modificados e incorporados en poemas anteriores; Ovidio, por tanto, podía pensar que ya el conjunto de su obra, y dentro de ella III 9, testimoniaban suficientemente su aprecio y su deuda con Tibulo. La segunda, que habiendo podido Ovidio escribir su poema completamente en el estilo de Tibulo, el que no lo haya hecho puede traslucir una delicadeza por su parte: lo que pretendía era rogar por él, no rivalizar con él.

Así, pues, ni Taylor, ni Munari parecen haber tomado conciencia de este sutil lazo que Ovidio da la impresión de haber querido tender entre su elegía y las de Tibulo: potenciar casi hasta la exageración uno de los rasgos que más caracterizaban los pentámetros del poeta difunto frente a los de los demás.

${ }^{4}$ Cf. también II 17, 16 concubuisse y III 7, 76 procubuisse; Pont. IV 10, 14 concubuisse.

${ }^{5}$ Para estas y otras semejanzas entre los versos de III 9 y Tibulo cf. F. Munari, P. Ovidi Nasonis Amores, testo, introduzione, traduzione e note, Florencia $1970_{5}$, notas ad loc. 
4. Para valorar este peculiar uso de formas verbales pentasilábicas (- $\cup-$ $\checkmark)$ terminadas en -isse $(t)$ hay que empezar teniendo en cuenta las posibles ubicaciones de dicho tipo de palabras en el esquema métrico del hexámetro y del pentámetro; luego hay que comparar la concurrencia con otros pentasílabos de estructura prosódica similar.

4.1. En cuanto a lo primero, hay que reconocer que en el pentámetro una palabra de forma $-\cup \cup \cup$, tiene, en principio, cabida tanto al comienzo del primer hemistiquio como del segundo. Ahora bien, si se emplea en el primero, conlleva ante la juntura o bien dos monosílabos o bien una palabra bisílaba, dos estructuras verbales de poco éxito en dicha posición (la segunda supondría un paralelismo total con el final de verso, cosa que se evita a medida que se va afianzando el bisílabo final).

En el hexámetro pueden tales palabras ubicarse en cinco lugares, haciendo coincidir su comienzo con el de los pies primero (A12B3) ${ }^{6}$, segundo (B34C5), tercero (C56X7), cuarto (X78Y9) o quinto (Y90Zz).

Virgilio las usa preferentemente a comienzo de verso, es decir, en A12B3; en segundo lugar (aunque con una frecuencia mucho menor que en el anterior), en X78Y9.

En final de hexámetro $(\mathrm{Y} 90 \mathrm{Zz})$ se las rehúye: aparecen 309 veces $(4,17$ por $100)$ en Lucrecio; dos veces $(0,09$ por 100) en Virgilio, Geórgicas y trece $(0,13$ por 100) veces en Virgilio, Eneida; dos veces en Ovidio, Metamorfosis $(0,02$ por 100).

En los hexámetros de los autores y obras que venimos analizando hemos encontrado estas palabras en posición final sólo tres veces $(0,92$ por 100$)$ en Catulo ${ }^{7}$, una $(0,28$ por 100$)$ en Propercio I y otra $(0,25$ por 100$)$ en Propercio II $^{8}$. En posición inicial aparece en Catulo una sola vez $(0,30$ por 100$){ }^{9}$; en Propercio, una en el libro primero $(0,28$ por 100$)$, tres en el tercero $(0,60$ por $100)$ y tres en el cuarto $(0,63$ por 100$)$, es decir, siete veces en total $(0,35$ por 100) ${ }^{10}$.

4.2. En lo que se refiere al segundo hemistiquio del pentámetro, además de los del Tipo A (surripuisse - $t$-) que venimos estudiando, se encuentran otros pentasílabos que hemos clasificado del siguiente modo:

\footnotetext{
${ }^{6}$ Según el sistema de símbolos propuesto por L. Nougaret («Une méthode de dépouillement destinée aux index métriques», $R E L 40,1962$, pp. 136-141).

7 68, 89; 68, 105; 97, 5; cinco veces según F. Cupaiuolo (Studi sull 'esametro di Catullo, Nápoles 1965, p. 36).

${ }^{8}$ I 8,35 ; II 26, 15.

${ }^{9}$ Según Cupaiuolo, op. cit., p. 63.

10 Según V. Viparelli, L'esametro di Properzio. Rapporti con Callimaco, Nápoles 1986 , pp. 86 ss. y 96 ss.
} 
Tipo H: excruciare(t); dentro de él incluimos también casos de final más o menos homófono, como destituere o corripiare.

Tipo Q: perpetuumque, sollicitetue, es decir, un tetrasílabo $U$ seguido de enclítica. Tipo Z: todos los demás.

Damos a continuación la frecuencia (porcentajes) con que se presentan cada uno de ellos en los distintos autores estudiados:

$\begin{array}{llccccc} & & \text { A } & \text { H } & \text { Q } & \text { Z } & \text { Total } \\ \text { CAT. } & & & & & & \\ \text { TIB. } & \text { I } & 1,53 & 0,31 & 0,61 & 1,85 & 4,3 \\ & \text { II } & 6,41 & - & 3,46 & 2,96 & 12,83 \\ & \text { I-II } & 6,05 & - & 6,05 & 1,40 & 13,50 \\ & \text { III } & 6,29 & - & 4,35 & 2,42 & 13,06 \\ & \text { IV } & 1,38 & 0,68 & 5,52 & 2,07 & 9,65 \\ \text { ProP. } & \text { I } & 4,40 & - & 5,49 & - & 9,89 \\ & \text { II } & 0,28 & 0,57 & - & 0,15 & 1,00 \\ & \text { III } & 0,88 & 0,15 & 0,15 & - & 1,18 \\ & \text { IV } & 1,21 & 0,80 & 0,20 & 1,01 & 3,22 \\ & \text { Total } & 0,84 & 2,10 & - & 0,21 & 3,15 \\ \text { Ov. } & \text { Am. I } & 1,85 & 0,85 & 0,10 & 0,35 & 2,15 \\ & \text { Am. II } & 3,20 & 1,04 & 1,04 & 3,12 & 7,02 \\ & \text { Am. III } & 4,59 & 2,07 & 2,22 & 0,98 & 7,14 \\ & \text { Total } \text { Am. } & 3,26 & 1,39 & 2,04 & 2,99 & 12,64 \\ & \text { Fast. I } & 1,93 & 0,83 & 4,97 & 4,14 & 11,06 \\ & \text { Pont. IV } & 1,08 & 0,43 & 2,81 & 3,25 & 7,59 \\ & \text { Total } & 2,54 & 1,07 & 2,73 & 2,88 & 9,22 \\ \text { MART. } & \text { I } & 1,61 & - & 2,82 & & 15,7211 \\ & \text { VI } & 2,02 & 0,50 & - & & 11,12 \\ & \text { XII } & 0,53 & - & 3,70 & & 11,64 \\ & \text { Total } & 1,42 & 0,16 & 2,20 & & 11,34\end{array}$

Ante estos datos es fácil reconocer que Tibulo I-II en conjunto, si bien alcanza porcentajes altos, no se diferencia tanto de los demás versificadores como lo hacía en el tipo A. Hasta con Propercio, que se muestra el más reacio a todos estos pentasílabos, se acortan un poco las distancias. Amores III es también aquí la obra que más se acerca a Tibulo. Marcial, por su parte, lo supera, sobre todo, en el libro I, como acabamos de decir, por su especial inclinación a los pentasílabos del tipo $\mathrm{Z}^{12}$ y dentro de ellos, muy en especial, a los vocativos como Caeciliane, Mamuriane, etc.

\footnotetext{
1 Datos estos, los generales, tomados de Giarratano 1905 y que suponen una alta frecuencia del tipo $\mathrm{Z}$.

12 Una tendencia esta de Marcial que podría relacionarlo en cierto modo con Catulo, quien, aunque en mucho menor grado, también muestra alguna insistencia en estos otros pentasílabos del tipo $\mathrm{Z}$.
} 
Por tanto, el rasgo típico de Tibulo no son los pentasílabos penúltimos del pentámetro, en general; tampoco lo son los pentasílabos verbales, ni siquiera los que hemos agrupado dentro del tipo $\mathrm{H}$ (excruciare $(t)$, destituere, corripiare). Lo que caracteriza a Tibulo, hasta el punto de constituir una especie de muletilla, son únicamente los pentasílabos terminados en -isse $(t)$, y muy en especial los infinitivos de perfecto ${ }^{13}$.

Con la única posible excepción de paenituisse (Tib. IV 12,4), cuya etimología no es clara, todos los demás son verbos compuestos de preposición, casi siempre monosilábica (continuisse, inseruisse, perdomuisse, secubuisse, etc.) y en una ocasión (praeteriisse: Tib. I 4, 34) disilábica.

A tales pentasílabos sólo serían comparables en frecuencia los del tipo Q, es decir, los constituidos por tetrasílabo $-\cup \cup$ más enclítica (composuitque, uerberibusque) que, como se puede comprobar, representan otra de las preferencias de Tibulo, si bien menos marcada que la anterior ${ }^{14}$.

Plessis ${ }^{15}$ entre los pentámetros con final disilábico reconoce varios tipos según la distribución de las palabras anteriores, entre los cuales ocupa el tercer lugar en frecuencia (después del tipo A, iugera multa soli, y el B cogor habere deos) el tipo $\mathrm{C}$, consuluisse uia, que ahora estudiamos, y da los siguientes datos al respecto en los distintos autores (porcentajes sobre el total de pentámetros con final bisilábico): Catulo, 13 por 100; Tibulo, 15 por 100; Propercio I, 2 por 100; Propercio IV, 3 por 100; Ovidio, Amores, 7,5 por 100; Tristia, 15 por 100. Ante estos datos concluye: «La forme $\mathrm{C}$ est particulièrement aimée de Tibulle d'Ovide (dans la seconde partie de son oeuvre élégiaque)... et aussi bien qu'un peu moins de Catulle. Au contraire Properce en use à peine».

Clarke ${ }^{16}$ revisó los datos de Plessis, proponiendo las siguientes cifras: Catulo, 14 por 100; Tibulo, 14,5 por 100; Propercio I, 2,2 por 100; IV, 2,9 por 100; Ovidio, Amores, 11,5 por 100; Heroides, 10,5 por 100; Tristia, 13,6 por 100. Afirmó, en consecuencia, que no existe diferencia considerable entre el primer Ovidio y el de las últimas obras; que en Catulo hay que tener en cuenta que sólo algo más de un tercio de los pentámetros terminan en disílabo y que,

${ }^{13}$ Sin que se den en él formas como conscelerasset (Cat. 67, 8) o sollicitasse (Ov. Am. I 7,14$)$ que en nuestro recuento hemos contabilizado también dentro del tipo A.

${ }^{14}$ Esto ya lo anotó en su día M. L. Clarke, que escribía ( A note on the augustan pentameter», Class. Rev. 61, 1947, p. 87, nota 3): "Tibullus is also much addicted to four-syllable words with -que added in this part of the pentameter. Such words are found in 33,7 per cent of the pentameters of the type under consideration; corresponding figures are: Propertius, 3,6 per cent; Ovid, Amores, 17,8 per cent; Tristia, 32,2 per cent. (figures include the few words with -ue added)").

15 F. Plessis, Traité de métrique grecque et latine, París 1889, p. 126 s.; y con él quienes se han ocupado de la tipología verbal del segundo hemistiquio del pentámetro.

16 Op. cit., p. 87. 
por tanto, no se puede comparar sin más con los otros versificadores. Propercio, como decía Plessis, recurre muy poco a esta combinación (además casi una cuarta parte de esos pentasílabos suyos son palabras griegas; mientras que dichas palabras en Tibulo no se dan, en Ovidio Amores suponen un 2,8 por 100 y en Tristia un 1,7 por 100). Está, pues, claro que Propercio evita los pentasílabos en esta posición, mientras que Tibulo y, en menor grado, Ovidio gustan de ellos.

Además, añade Clarke, en Tibulo un 45,7 por 100 de tales pentasílabos son infinitivos de perfecto. En cambio, en Propercio lo son sólo un 28,5 por 100; en Amores, un 25 por 100 y en Tristia un 23,4 por 100.

De suyo, los pentasílabos del tipo $\cup \cup \cup$ no son muy frecuentes en latín y una buena cantera la proporcionaban estos infinitivos de perfecto. A veces incluso, anota Clarke ${ }^{17}$, vemos a Tibulo recurrir a ellos cuando la sintaxis pedía más bien un infinitivo de presente:

quam iuuat immites uentos audire cubantem

'et dominam tenero continuisse sinu ${ }^{18}$,

de modo que se puede concluir «that with Tibullus the use of the perfect infinitive in this position was rather like Cicero's esse uideatur, a cliché employed to secure a favourite rhythm».

A la hora de preguntarse por el significado de la diferencia detectada en este punto entre los poetas y por el sentido de un final como consuluisse uia en medio de los demás posibles, Clarke recuerda las observaciones de Quintiliano sobre la estructura verbal de las cláusulas de la prosa o del hexámetro ${ }^{19}$. Y, aun teniendo en cuenta que aquí no se trata propiamente de un final ${ }^{20}$, concluye: «But a pentameter ending such as consuluisse uia would surely be felt to move more swiftly than one which was broken up into a greater number of words; and it may be that to a sensitive ear it would not be altogether free from the taint of mollitia. We may say that Tibullus was consciously aiming at a swift-moving and smooth ending to the pentameter, while Propertius definitely avoided such an effect».

17 Op. cit., p. 87.

18 Tib. I 1, 45.

19 Quintiliano, teniendo en cuenta el latens tempus existente en la diuisio uerborum (IX 4, 98), calificaba de forte una cláusula como criminis causa y de molle otra como archipiratae (IX 4, 97) e igualmente rechazaba como praemolle un final de hexámero como fortissima Tyndaridarum.

20 Aunque, añadimos nosotros, no hay que olvidar el carácter de cláusula que tiene todo este segundo hemistiquio, que cierra no sólo el pentámetro, sino también el dístico. Y además, como veremos en seguida, no se descarta, en nuestra opinión, una cierta correspondencia entre este grupo de cinco sílabas $-\cup \cup$ que precede al bisílabo último y la cláusula del hexámetro. 
5. Una vez detectada y comprobada esta tendencia y una vez vislumbrado el posible sentido que se le puede reconocer, procede intentar delimitarla y precisarla un poco más. Para lo cual hemos rastreado también otros tipos de combinaciones de palabras que en dicha posición del pentámetro, es decir, ante el bisílabo final, podían dar una secuencia $\cup \cup \cup$ lo más parecida al tipo $\mathrm{A}$, tanto en lo que respecta a tipología verbal como en lo que toca a factores léxicos, gramaticales e incluso fónicos, o sea, con una cadencia -isse $(t) o$ -esse $(t)$. En ese sentido, hemos distinguido los siguientes:

Tipo B: - | $\cup \cup$, non habuisse(t)

Tipo C: $\cup \mid \cup \sim$, nulla dedisse $(t)$, corpor(a abesse $(t)$

Tipo D: $\smile \cup \mid-\cup$, ultima nosse $(t)$, desiner(e esse $(t)$

Tipo E: - $|\cup| \smile$, non tamen esse(t), me memin(i esse $(t)$

Tipo F: $\sim|\cup| \sim$, saepe quod esse(t).

He aquí los resultados del recuento:

\begin{tabular}{|c|c|c|c|c|c|c|c|c|}
\hline & & A & B & $\mathrm{C}$ & D & $E$ & $F$ & Total \\
\hline CAT. & & 1,53 & - & 0,61 & 1,54 & 0,31 & 0,31 & 4,3 \\
\hline Тів. & $\begin{array}{l}\text { I } \\
\text { II } \\
I-I I \\
\text { III } \\
\text { IV }\end{array}$ & $\begin{array}{l}6,41 \\
6,05 \\
6,29 \\
1,38 \\
4,40\end{array}$ & $\begin{array}{l}2,72 \\
0,93 \\
2,10 \\
\overline{4,39}\end{array}$ & $\begin{array}{l}0,99 \\
0,46 \\
0,81 \\
2,07 \\
3,30\end{array}$ & $\begin{array}{c}1,73 \\
0,46 \\
1,29 \\
1,4 \\
-\end{array}$ & $\begin{array}{l}0,74 \\
0,93 \\
0,81 \\
-\overline{2,20}\end{array}$ & $\begin{array}{l}- \\
- \\
-\end{array}$ & $\begin{array}{r}12,59 \\
8,83 \\
11,30 \\
4,85 \\
14,29\end{array}$ \\
\hline Prop. & $\begin{array}{l}\text { I } \\
\text { II } \\
\text { III } \\
\text { IV } \\
\text { Total }\end{array}$ & $\begin{array}{l}0,28 \\
0,88 \\
1,21 \\
0,84 \\
0,85\end{array}$ & $\begin{array}{l}0,85 \\
1,61 \\
1,21 \\
0,21 \\
1,05\end{array}$ & $\begin{array}{l}0,28 \\
0,73 \\
1,42 \\
1,47 \\
0,99\end{array}$ & $\begin{array}{l}1,98 \\
2,35 \\
1,82 \\
1,68 \\
1,99\end{array}$ & $\begin{array}{l}0,28 \\
2,06 \\
1,22 \\
1,68 \\
1,47\end{array}$ & $\begin{array}{l}- \\
- \\
-\end{array}$ & $\begin{array}{l}3,67 \\
7,63 \\
6,88 \\
5,88 \\
6,35\end{array}$ \\
\hline Ov. & $\begin{array}{l}\text { Am. I } \\
\text { Am. II } \\
\text { Am. III } \\
\text { Total Am. } \\
\text { Fast. I } \\
\text { Pont. IV } \\
\text { Total }\end{array}$ & $\begin{array}{l}1,82 \\
3,20 \\
4,59 \\
3,26 \\
1,93 \\
1,08 \\
2,54\end{array}$ & $\begin{array}{l}0,52 \\
0,49 \\
0,46 \\
0,49 \\
\overline{1,08} \\
0,54\end{array}$ & $\begin{array}{l}1,56 \\
2,71 \\
1,40 \\
2,04 \\
1,10 \\
1,08 \\
1,66\end{array}$ & $\begin{array}{l}1,04 \\
2,71 \\
2,30 \\
2,04 \\
0,55 \\
4,55 \\
2,34\end{array}$ & $\begin{array}{l}- \\
0,49 \\
0,92 \\
0,50 \\
0,28 \\
2,38 \\
0,88\end{array}$ & $\begin{array}{l}-\overline{0,27} \\
0,23 \\
0,16 \\
0,28 \\
-15\end{array}$ & $\begin{array}{r}4,94 \\
9,87 \\
9,90 \\
8,49 \\
4,14 \\
10,17 \\
8,11\end{array}$ \\
\hline MART. & $\begin{array}{l}\text { I } \\
\text { VI } \\
\text { XII } \\
\text { Total }\end{array}$ & $\begin{array}{l}1,61 \\
2,02 \\
0,53 \\
l, 42\end{array}$ & $\begin{array}{l}0,40 \\
0,50 \\
0,53 \\
0,47\end{array}$ & $\begin{array}{l}0,40 \\
1,01 \\
0,53 \\
0,63\end{array}$ & $\begin{array}{l}2,01 \\
1,01 \\
\frac{1,10}{}\end{array}$ & $\begin{array}{l}0,81 \\
1,52 \\
4,23 \\
2,08\end{array}$ & $\begin{array}{l}0,40 \\
0,50 \\
1,06 \\
0,64\end{array}$ & $\begin{array}{l}5,63 \\
6,56 \\
6,88 \\
6,34\end{array}$ \\
\hline
\end{tabular}

También por esta otra vía se perfila la tendencia de Tibulo: su inclinación no es tanto por las terminaciones -isse, -esse ante el bisílabo final del pentámetro, cuanto por los pentasílabos con dicha cadencia, es decir, por los infinitivos de perfecto. Lo más próximo a dichos pentasílabos, la combinación de monosílabo más tetrasílabo del tipo B (una estructura prosódica muy similar 
a la de los verbos del tipo A, constituidos casi siempre, como hemos dicho, a base de preverbio monosilábico), es también lo que les sigue en frecuencia. Pero este otro tipo ya no parece característico suyo en exclusiva; se usa el doble de veces en el libro IV ${ }^{21}$.

6. He aquí, por tanto, un rasgo curioso de la lengua y de la versificación de Tibulo. Un rasgo que Ovidio supo captar y que tuvo la sutil habilidad de utilizar cuando se propuso rendir homenaje al poeta elegíaco.

Cabría aún preguntarse si estas formas verbales en tal posición del dístico elegíaco, junto a los otros tipos que hemos manejado y todo ello unido a otros fenómenos de tipología verbal, de distribución de sonidos o de sintaxis y fraseología que parecen detectarse en el mismo sitio, podrían responder a una atención especial prestada por los versificadores a la secuencia $-\cup \cup \cup \cup$ que precede al bisílabo final del pentámetro; una atención que respondería al sentimiento (consciente o inconsciente) de una cierta correspondencia entre ella y la cadencia final del hexámetro $(-\cup \cup-X)$ : no se olvide que en ambos casos se trata del mismo esquema cuantitativo fijo y que en ambos se da la misma tendencia a la acomodación de acentos y tiempos marcados. Pero todo ello, y la posible relación que pueda tener con el progresivo predominio de bisílabos finales en el pentámetro latino, es algo que merece tanta prudencia como estudio detenido, un estudio que dejamos aplazado para otra ocasión.

JESÚS LUQUe MORENO

\section{Bibliografia}

Clarke, M. L., 1947: «A note on the augustan pentameter», Class. Rev. 61, pp. 86-88. Cupaiuolo, F., 1965: Studi sull'esametro di Catullo, Nápoles.

Fraenkel, H., 1945 (=1956): Ovid. A poet between two worlds, Berkeley-Los Ángeles. Giarratano, C., 1908: De M. Val. Martialis re metrica, Nápoles.

McKeown, J. C., 1987: Ovid: Amores. Text, Prolegomena an Commentary, I-IV, Liverpool, New Hampshire.

Munari, F., 1970s: P. Ovidi Nasonis Amores, testo, introduzione, traduzione e note, Florencia.

Nougaret, L., 1962: «Une méthode de dépouillement destinée aux index métriques», REL 40, pp. 136-141.

Plessis, F., 1869: Traité de métrique grecque et latine, París.

Perret, J., 1954: «Mots et fins de mots trochaïques dans l'hexamètre latin», REL 32, pp. 182-199.

${ }^{21}$ «It should be said -escribía Clarke, op. cit., p. 87, nota 4- that in pentameter endings of Plessis's type E (mors adoperta caput), for which Tibullus shows no particular predilection, the proportion of perfect infinitives is also high. The figure, 28 per cent., is, however, noticeably lower than that for type $\mathrm{C}$ \%. 
Taylor, J. H., 1970: «Amores 3, 9: A Farewell to Elegy», Latomus 29, pp. 474-477. Thomas, E., 1965: «Ovid, Amores 3, 9, 35-40», Classical Review n.s. 15, pp. 149-151. Thomas, E., 1965: "A comparative Analysis of Ovid Amores 2, 6 and 3, 9», Latomus 24, pp. 599-609.

Viparelli, V., 1986: L'esametro di Properzio. Rapporti con Callimaco, Nápoles. 